OPEN ACCESS

Edited by:

Xiaomin $\mathrm{Li}$

Fudan University, China

Reviewed by:

Ruoxue Yan,

University of California, Riverside,

United States

Xiaoji Xie,

Nanjing Tech University, China

Yiqing $L u$,

Macquarie University, Australia

${ }^{*}$ Correspondence:

Yao He

yaohe@suda.edu.cn

${ }^{\dagger}$ These authors have contributed equally to this work

Specialty section

This article was submitted to

Nanoscience,

a section of the journal

Frontiers in Chemistry

Received: 06 December 2017

Accepted: 14 February 2018

Published: 28 February 2018

Citation:

Ji X, Wang H, Song B, Chu B and He $Y$ (2018) Silicon Nanomaterials for Biosensing and Bioimaging Analysis.

Front. Chem. 6:38.

doi: 10.3389/fchem.2018.00038

\section{Silicon Nanomaterials for Biosensing and Bioimaging Analysis}

\author{
Xiaoyuan $\mathrm{Ji}^{\dagger}$, Houyu Wang ${ }^{\dagger}$, Bin Song ${ }^{\dagger}$, Binbin Chu and Yao He* \\ Laboratory of Nanoscale Biochemical Analysis, Institute of Functional Nano and Soft Materials and Jiangsu Key Laboratory \\ for Carbon-Based Functional Materials and Devices, Collaborative Innovation Center of Suzhou Nano Science and \\ Technology, Soochow University, Suzhou, China
}

Biochemical analysis in reliable, low-toxicity, and real-time manners are essentially important for exploring and unraveling biological events and related mechanisms. Silicon nanomaterial-based sensors and probes have potentiality to satisfy the above-mentioned requirements. Herein, we present an overview of the recent significant improvement in large-scale and facile synthesis of high-quality silicon nanomaterials and the research progress of biosensing and bioimaging analysis based on silicon nanomaterials. We especially illustrate the advanced applications of silicon nanomaterials in the field of ultrasensitive biomolecular detection and dynamic biological imaging analysis, with a focus on real-time and long-term detection. In the final section of this review, we discuss the major challenges and promising development in this domain.

Keywords: silicon, nanomaterials, synthesis, biosensing, bioimaging

\section{INTRODUCTION}

During the past decades, functional nanomaterials [e.g., fluorescent semiconductor quantum dots (QDs), graphene, carbon nanodots, gold/silver nanoparticles (Au/Ag NPs), etc.] have been intensively employed for the design of various biosensors and probes, owing to their excellent physicochemical properties (e.g., unique optical/electronic performance, large ratios of surface-to-volume, and good surface tailorability as well as abundant surface chemistry, etc.) (Jung et al., 2010; Holzinger et al., 2014; Tilmaciu and Morris, 2015). With the rapid development of silicon nanotechnology, silicon nanostructures/nanohybrids with attractive properties have been extensively developed for the rational fabrication of high-quality sensors and probes for bioimaging and biosensing applications (Nishimura et al., 2013; Wang et al., 2013; Lai et al., 2016). It is worth pointing out that silicon nanomaterials [e.g., silicon nanoparticles (SiNPs), silicon nanoneedles] could easily biodegrade into renal clearable molecules (i.e., silicic acid) and then excrete out the body with no evidence of toxicity in vivo (Park et al., 2009; Chiappini et al., 2015). Of particular concern is that ultra-small (diameter: $3-10 \mathrm{~nm}$ ) Si NPs have received the Food and Drug Administration (FDA)-approved investigational new drug approval for first-in-human clinical trials (Phillips et al., 2014). Consequently, different dimensional silicon nanomaterials have been prepared and functionalized for various analytical applications. For instance, zero-dimensional fluorescent SiNPs featuring good water-dispersibility, strong fluorescence, as well as ultrahigh photostability, have been proved to be ideally suitable for tracking live cells in real-time and long-term ways (Peng et al., 2014; Zhong et al., 2015). On the other side, one-dimensional silicon nanowires (SiNWs) and two-dimensional silicon wafer nanohybrids (e.g., silicon wafer decorated with metal NPs) could be designed as a general biosensing technology for enhanced surface-enhanced Raman scattering (SERS) studies (Wipf et al., 2013; Wang et al., 2016). Compared 
with free metal NPs-based SERS sensors, silicon-based SERS sensors embody superior sensitivity and reproducibility. Consequently, various kind of functional silicon SERS sensors have been exploited for sensitively and selectively detecting myriad biological and chemical species in reliable and reproducible manners.

Herein, this review article will briefly summarize recent significant improvement in the preparation of silicon nanomaterials and their bioapplications in biochemical analysis (Scheme 1). In the following sections, we first present recent efforts in preparing fluorescent silicon nanomaterials with high luminescence in facile and large-scale manners. Then, we illustrate the typical examples of sensors and bioimaging analysis based on silicon nanomaterials. In the last section, we examine future challenges and potentialities associated with myriad biochemical analysis studies based on silicon nanomaterials.

\section{SYNTHESIS OF SILICON NANOMATERIALS}

Since the first discovery of the unique optical properties of fluorescence silicon nanomaterials (Wilson et al., 1993; Park et al., 2001), numerous synthetic strategies have been reported to prepare silicon nanomaterials with high fluorescence and photostability, vastly facilitating the exploration of their optical applications in biochemical analysis (Atkins et al., 2011; Zhou et al., 2015; Dasog et al., 2016; Liu et al., 2016). Combined with current research concerns, this section intends to briefly summarize recent progresses in facile and large-quantity synthesis of fluorescence silicon nanomaterials, including zerodimensional SiNPs and one-dimensional silicon nanostructures.

Various high-efficacy and workable strategies have been introduced for the large-scale preparation of fluorescence SiNPs with strong fluorescence [photoluminescent quantum yield (PLQY): $\sim 15-25 \%$ ] and robust photostability in facile and rapid manners, which are fundamentally critical for their long-awaited applications. By virtue of fast rise of temperature and uniform heating of samples, microwave irradiation is advantageous for large-scale and rapid synthesis of high-quality fluorescent SiNPs. As a typical example, Zhong et al. reported that $0.1 \mathrm{~g}$ SiNPs could be readily obtained within 10 min by using organosilicon molecules as silicon precursors via microwave-assisted method (Zhong et al., 2013). Recently, by using low-cost and nontoxic silicon resources (e.g., rice husks, wheat straws, and diatoms, etc.), a microwave-assisted biomimetic method was further developed for synthesizing SiNPs in an environmentally friendly manner (Wu S. C. et al., 2015; Wu et al., 2016). Besides aforementioned microwave equipment-assisted strategy, a photochemical method has gained researchers' attention, which can be used to prepare SiNPs in glass flasks under mild conditions (i.e., room temperature and normal pressure) (Zhong et al., 2015). Of particular note, $\sim 10 \mathrm{~g}$ high-quality SiNPs could be obtained in short time $(<40 \mathrm{~min})$ under UV irradiation using a potable xenon lamp, which sufficiently satisfied the need of wide-ranging biological applications.

Different from zero-dimensional SiNPs, one-dimensional fluorescent silicon nanostructures possess unique optical properties (Zheng et al., 2012; Wu K. F. et al., 2015). Particularly, reduced thresholds of multiexciton generation and optical gain of one-dimensional silicon nanostructures are beneficial for the fabrication of high-performance silicon nanomaterialbased nanolasers and nanodevices (Shabaev et al., 2013). The pioneering example of one-dimensional fluorescent silicon nanorods (SiNRs) has been reported in 2013 (Lu et al., 2013). In this work, relative low luminescence (PLQY: 5\%) SiNRs have been prepared by the decomposition of trisilane in hot squalane with the presence of Tin NPs and odecylamin, followed by hydrogen fluoride (HF) and thermal treatment. Recently, on the basis of microwave-assisted synthetic approach for preparing SiNPs (Zhong et al., 2013), highly luminescent SiNRs with PLQY of $\sim 15 \%$ have been further fabricated by adding milk into the reaction precursors (Song et al., 2016). Briefly, crystal nucleation including silicon and carbon nanoclusters could be firstly created through microwave irradiation. Meanwhile, calcium phosphate (Cap) crystallization was formed through fusion-fission between $\mathrm{Ca}$ or $\mathrm{P}$ ions linked protein micelles in the presence of aminosilane, which facilitated the aggregation of the silicon and carbon nuclei, resulting in one-dimensional silicon nanomaterials with rod-structures. Particularly, the as-prepared SiNRs possessed excitation wavelength-dependent fluorescence spectra and have been conceptually developed for the construction of white-light-emitting devices (LEDs). Lately, the same group introduced a new type of one-dimensional multifunctional silicon shuttles (SiNSs), which could be obtained by addition of $\mathrm{Fe}^{3+}$ ions into the same silicon source. Following the above-mentioned workflow, SiNSs were fabricated through $\mathrm{Fe}^{3+}$-induced oriented attachment mechanism (Song et al., 2017). Significantly, the resultant SiNSs featuring intrinsic magnetism and excitation-wavelength dependant luminescence simultaneously were proved to be superbly suitable for advanced anti-counterfeiting application with additional magnetismrelated secrecy (Song et al., 2018). As thus, benefiting from advantages of microwave, the presented strategies have been proved to be efficient and general synthetic approaches for preparing one-dimensional silicon nanomaterials rapidly and facilely; and moreover, such method shows great promise for developing fluorescent silicon nanomaterials with multiple functionalities.

\section{BIOSENSING}

The past decade has witnessed the exciting achievements in the fields of silicon nanomaterials/nanohybrids-based sensors, enabling determination of myriad biological and chemical species in sensitive and reliable manners. In this section, we focus on introducing typical recent advances of siliconbased biosensors, particularly including field-effect transistor (FET) sensors, fluorescent sensors and surface-enhanced Raman scattering (SERS) sensors.

The FET sensors can evidently amplify electronic signals, which are mainly composed of a semiconductor path (defined as "channel") and two electrodes (defined as "source" and "drain," respectively) (Knopfmacher et al., 2014). Specifically, the 


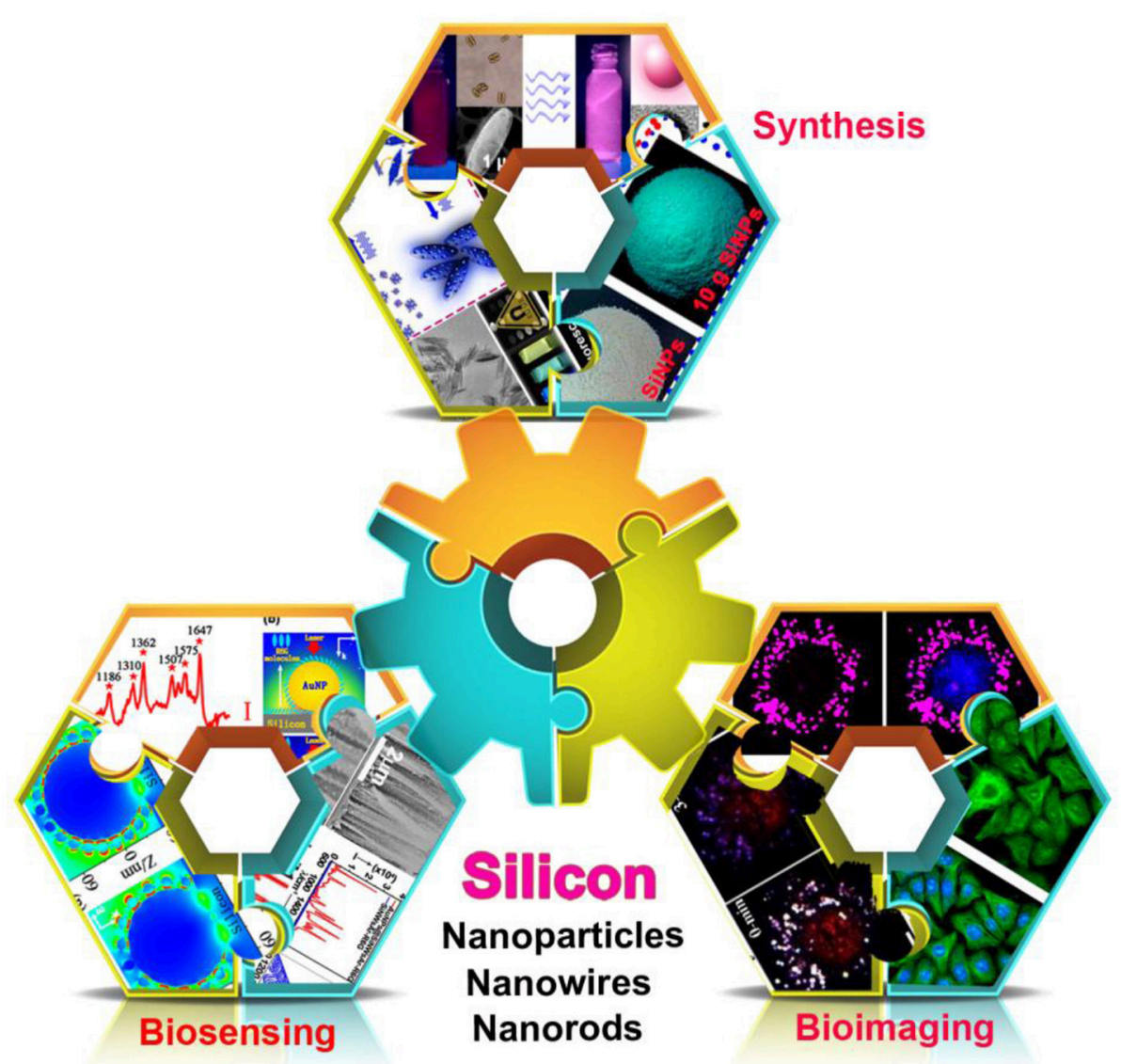

SCHEME 1 | Fabrication of silicon nanomaterial-based platform for biochemical applications [i.e., synthesis of silicon nanomaterials (Wu S. C. et al., 2015) (reprinted with permission, Copyright 2015, ACS Publications); (Zhong et al., 2015) (reprinted with permission, Copyright 2015, ACS Publications); (Song et al., 2016) (reprinted with permission, Copyright 2016, ACS Publications), biosensing (Sun et al., 2015) (reprinted with permission, Copyright 2015, ACS Publications); (Wang et al., 2014) (reprinted with permission, Copyright 2014, AIP publications); (Zhu et al., 2015) (reprinted with permission, Copyright 2015, ACS Publications), and bioimaging (Zhong et al., 2013) (reprinted with permission, Copyright 2013, ACS Publications); (Wu S. C. et al., 2015) (reprinted with permission, Copyright 2015, ACS Publications); (Ji et al., 2015) (reprinted with permission, Copyright 2015, Wiley-VCH)].

conductance signals of FET sensors could vary when detecting biological or chemical species, which would induce a negative or positive gate voltage. To date, SiNWs-based FET sensors have been used for real-time, label-free, sensitive, and multiplexed determination of a variety of species, including chemical reagents as well as biological species [e.g., sodium ions (Wipf et al., 2013), nucleic acids (Gao et al., 2013; Lu et al., 2014), cancer biomarker (Shehada et al., 2014), and proteins (Krivitsky et al., 2016) etc.]. Recently, Krivitsky et al. reported a simple and efficient strategy for sensing specific biomarkers directly from unprocessed biosamples using antibody-modified SiNW-based FET devices, which was free of time-consuming manipulation procedures (Krivitsky et al., 2016). As illustrated in Figure 1A (left panel), when the biosample contained analyte biomarker, the specific binding of biomarker on antibody-modified SiNW FET device would result in relative slower rate of returning to baseline during the "dissociation regime" compared to those of control groups (e.g., SiNW modified with non-specific antibody or no antigen in the sample). Based on the presented sensitive and selective approach, the mouse monoclonal antihuman cancer 15-3 IgG (CA 15-3) in practical sample was readily detected and quantified by using the anti-CA 15-3-modified SiNW FET sensing device based on the corresponding dissociation kinetic curves (Figure 1A, right panel). In addition to the conventional affinity-based FET sensors, C. Lieber's group also developed several types of SiNWs-based transistors for recording neural activity in multiplexed, long-term, and high-resolution manners (Qing et al., 2014; Xie et al., 2015). For example, they stereotaxically implanted the 3D mesh-based nanoelectronics incorporated with SiNWs-based FET sensors in a frozen state into rodent brains with minimal damage, and employed it to record multiplexed local field potentials (LFPs) and single-unit action potentials from the somatosensory cortex, opening up new avenues for implantation and long-term brain activity mapping based on silicon nanomaterials (Xie et al., 2015).

Fluorescent sensors feature excellent sensitivity, short-time data acquisition, facile manipulations and low cost, which have been intensively explored for a myriad of sensing 



FIGURE 1 | (A) Schematically illustrating the workflow of antibody-conjugated SiNW-based FET sensor device (upper part, green receptor units) vs. non-immune reactive protein-conjugated control device (lower part, red receptor units) (left panel). Right panel: Specific (anti-CA 15-3) and non-specific protein (BSA CA-15-3) functionalized SiNW-based FET sensor devices for the detection of the CA 15-3 antigen. Reprinted with permission from Krivitsky et al. (2016). Copyright (2016) ACS Publications. (B) Eu@SiNRs for intracellular pH measurement. Confocal images of internalized Eu@SiNRs in MCF-7 cells with different cytoplasmic pH values (i.e., 3-9) (left panel). Scale bars $=25 \mu \mathrm{m}$. Right panel: Corresponding histograms of the fluorescence intensity ratio $\left(R=I_{470} / /_{620}\right)$ vs. $\mathrm{pH}$ values ranging from 3 to 9. Inset is the linear relationship between $R$ and pH values (i.e., 4-9). Reprinted with permission from Chu et al. (2017). Copyright (2017) ACS Publications.

applications. By virtue of strong and stable fluorescence of silicon nanomaterials, various types of fluorescent silicon nanomaterialbased sensors have been designed and fabricated for the detection of biological and chemical species, including glucose (Yi et al., 2013a), agricultural chemicals (Yi et al., 2013b), nitroaromatic explosives (Gonzalez et al., 2014; Ban et al., 2015), food additives (Jose et al., 2016), and intracellular $\mathrm{pH}$ (Chu et al., 2016, 2017), and so forth. Very recently, Chu et al. presented one dimensional europium (Eu)-doped SiNRsbased ratiometric sensing system without additional chemical modification, allowing for detecting intracellular $\mathrm{pH}$ fluctuation in live cells in real-time and long-term manners (Chu et al., 2017). Particularly, the presented Eu@SiNRs featured pHsensitive emission peak at $470 \mathrm{~nm}$ and $\mathrm{pH}$-insensitive one at $620 \mathrm{~nm}$ simultaneously under single-wavelength excitation, thus producing ratiometric signals $\left(R=I_{470} / I_{620}\right)$. Remarkably, the developed sensors exhibited broad-pH response (e.g., 3-9) in human breast cancer (MCF-7) cells (Figure 1B, left panel), which was confirmed by corresponding liner regression equation and correlation coefficient (Figure 1B, right panel). The instinct fluorescence emission change of Eu@SiNRs probe with pH fluctuation eliminated linking of $\mathrm{pH}$-sensitive moiety and further modification of reference fluorophores, providing novel strategies for facile fabrication of high-quality ratiometric sensors based on fluorescent nanomaterials.

SERS is able to amplify the feeble Raman intensity ideally up to $10^{14} \sim 10^{15}$, offering ultrasensitive avenues to explore the Raman signals at the single-molecule level. Compared to free Au NPs or Ag NPs-based SERS-active substrates, silicon nanohybrids (Au/Ag NPs-decorated silicon wafer or SiNWs array)-based SERS substrates feature superior SERS enhancement and better reproducibility (Shi et al., 2017). The distinct SERS enhancement is originated from the hybridization of metal nanoparticles-scattered electromagnetic field and Sireflected electromagnetic field (Wang et al., 2014). Meanwhile, the improved reproducibility is ascribed to uniform metal 
nanoparticles tightly anchored on the silicon surface, efficiently avoiding the uncontrollable aggregation of free nanoparticles in liquid phase (Wang et al., 2016). Taking advantages of these merits, silicon nanohybrids-based SERS substrates are ideally suitable for the analysis of myriad biological and chemical samples in practical systems in sensitive, reliable and reproducible manners, such as apoptotic cell (Jiang et al., 2013), mercuric ion (II) (Sun et al., 2015), DNA (Zhu et al., 2015), bacteria (Wang et al., 2015), lead ions (Shi et al., 2016), and trinitrotoluene (TNT) (Chen et al., 2017). On the basis of these exciting works, silicon nanohybrids-based SERS sensors have been well-designed as portable and reliable analytical platforms, which serve as powerful tools for tracing specific compound from environmental samples.

\section{BIOLOGICAL IMAGING}

SiNPs featuring benign biocompatibility and unique optical properties (i.e., strong fluorescence coupled with ultrahigh photostability) are emerging as novel high-quality fluorescent nanoprobes for biological imaging analysis, particularly for tracking dynamic biological procedures in long-term and realtime manners.

Systematic characterizations of bio-behaviors of SiNPs in biological systems (e.g., cellular internalization mechanism, intracellular trafficking, and final destination, etc.) are crucial for reliable toxicology analysis, providing a feasible evaluation of utilizing SiNPs for biological applications. By virtue of strong and stable fluorescent signals, the cellular behaviors of SiNPs could be dynamically monitored in live cells (Shiohara et al., 2010, 2011; Cao et al., 2017; Zhou et al., 2017). Very recently, comprehensive and reliable investigations of cellular internalization and intracellular fate of SiNPs have been revealed by analyzing the colocalization of SiNPs with various subcellular compartments (Cao et al., 2017). Typically, SiNPs were internalized into cells mainly through clathrinmediated and caveolae-dependent endocytosis and actively transported from periphery to the perinuclear region along microtubules after cellular internalization. Along with efficient
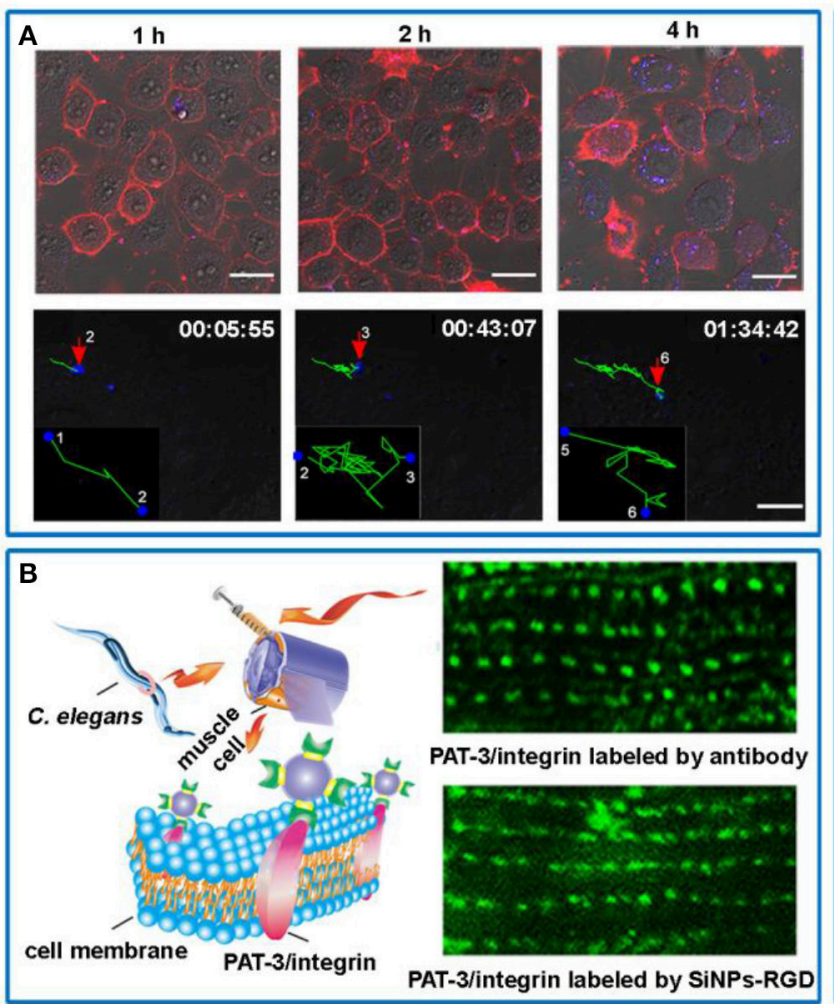

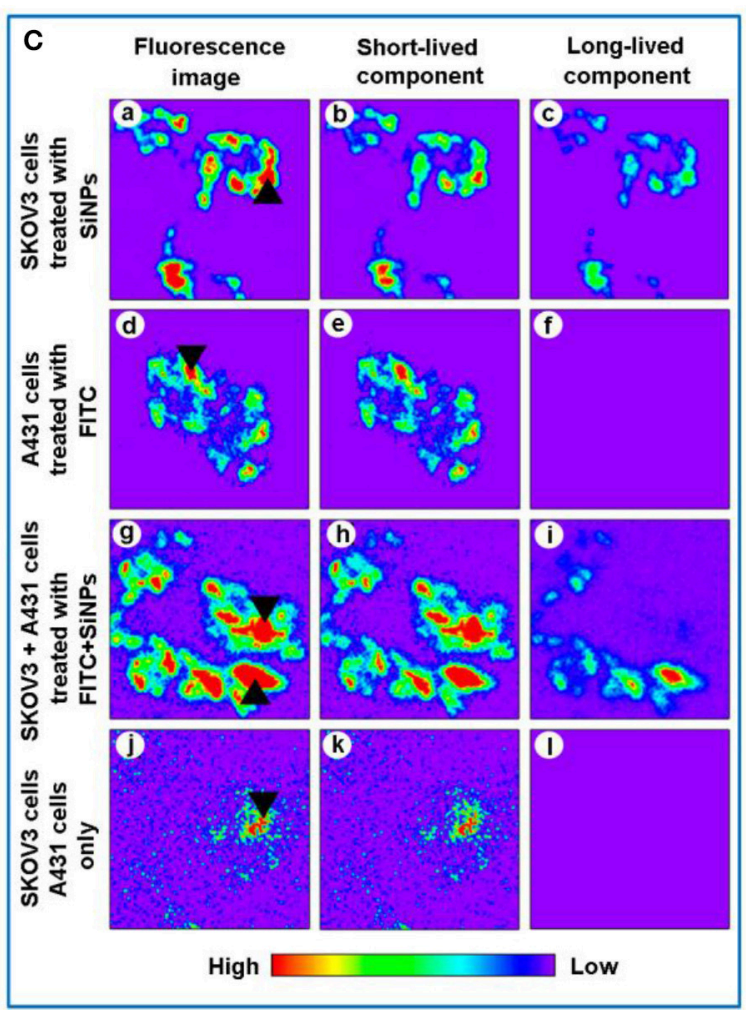

FIGURE 2 | (A) Confocal images of time-dependent cellular uptake of PEI-SiNPs/pDNA nanocomplexes in HeLa cells (top panel). Cell membranes were stained with Dil. Fluorescence of SiNPs and Dil is defined as blue and red, respectively. Scale bars $=20 \mu \mathrm{m}$. Bottom panel: Real-time and long-term tracking the dynamic movement of the nanocomplexes in a live cell. The movement trajectory is delineated in green line. Reprinted with permission from Pang et al. (2016). Copyright (2016) Springer. (B) Schematic illustration of SiNP-RGD for labeling PAT-3/integrin at the muscle cell membrane in C. elegans (left panel). Right panel: Specific labeling the subcellular PAT-3/integrin using SiNP-RGD and PAT-3 antibody. Reprinted with permission from Zhou et al. (2017). Copyright (2017) Springer. (C) Fluorescence images and time-gated confocal images of SKOV3 cells immunostained by anti-HER2-modified SiNPs (a-c), A431 cells stained by anti-mouse secondary antibodies-labeled FITC (d-f), co-cultured SKOV3 cells and A431 cells labeled by anti-HER2-modified SiNPs and FITC coupled with anti-mouse secondary antibodies, respectively (g-i), and non-treated SKOV3 cells and A431 cells (j-I). Reprinted with permission from Tu et al. (2017). Copyright (2017) ACS Publications. 
internalization, SiNPs showed no apparent toxic effect on cell growth, as demonstrated by the metabolic activity and integrity of the plasma membrane. Besides above-mentioned cellular investigations in vitro, visual observation of in vivo behaviors of SiNPs was further achieved, facilitating the extensive utilization of SiNPs for biological and biomedical applications (Zhou et al., 2017). By using C. elegans as a classic model organism, biodistribution, stability, and biocompatibility of SiNPs in live organisms have been elucidated. The distribution of SiNPs could be altered by different administration methods; and moreover, the internalized SiNPs would reserve in specific organisms without diffusion during long-term observation time (i.e., $4 \mathrm{~h}$ ), demonstrating the possibility of using SiNPs-based bioprobes for specific tissue imaging studies. In addition, SiNPs have a little or no toxic effect on body morphology, life span, and reproduction ability of tested worms, implying the superb biocompatibility of SiNPs in living organisms. These findings suggest the possibility for design of high-quality biocompatible SiNPs-based bioprobes for long-term and real-time tracking biological events in vitro and in vivo.

Biologically relevant molecules [e.g., transferrin (Tf) (Nishimura et al., 2013), sugar (Lai et al., 2016), targeting peptides (Song et al., 2015; Zhou et al., 2017), and polyethylenimine (PEI) (Pang et al., 2016), etc.] have been utilized to functionalize SiNPs, producing the SiNPs-based biofunctional nanoprobes for dynamically studying carbohygrate-carbohydrate interactions, single Tf receptor (TfR) molecule tracking, and targeting specific cancer cells. In particular, benefiting from the nonor low toxicity and high photostability of SiNPs, SiNPs-based fluorescent probes are superbly suitable to track cell-biological interactions in living cells in a real-time way, which have been studied by several groups. In 2016, Pang et al. developed PEI encapsulated SiNPs nanocomposites, which simultaneously possessed bright and stable fluorescence, high DNA-binding capacity ( $\sim 97 \%)$, and adaptable transfection efficiency $(\sim 35 \%)$ in human cervical carcinoma (HeLa) cells as well as feeble cytotoxicity (Pang et al., 2016). Taking advantages of these merits, the dynamic transport of internalized SiNPs-based carriers could be monitored by detecting stable and bright blue fluorescence signals of SiNPs (Figure 2A, top panel). The entire trajectory presented that SiNPs-based gene carriers moved toward perinuclear region, which was advantageous to efficiently delivery genetic information into nucleus (Figure 2A, bottom panel). As a typical example, by functionalizing SiNPs with cyclic RGD (i.e., arginine-glycine-aspartic acid) peptides, the resultant SiNPs-RGD bioprobes allowed for monitoring integrin-mediated endocytosis during persistent observation time ( $\sim 120 \mathrm{~min}$ ) (Song et al., 2015). Such SiNPs-RGD bioprobes were further proved to be suitable for specifically labeling and imaging of body-wall muscle cells in live C. elegans by targeting PAT-3/integrin at a molecular-level (Figure 2B) (Zhou et al., 2017). These works suggest that RGD functionalized SiNPs can be used as a general tool for in vitro and in vivo bioimaging analyses, which is also confirmed by cancer-related in vivo applications addressed by Erogbogbo et al. (2011) and Ji et al. (2015) independently.

It is worth noting that the imaging resolution of SiNPsbased fluorescence imaging can be dramatically improved by using time-gating techniques ( $\mathrm{Gu}$ et al., 2013; Joo et al., 2015; Kim et al., 2017; Tu et al., 2017). Early in 2013, Gu et al. utilized photoluminescent porous SiNPs (pSiNPs) with unusually long-emission lifetime $(5-13 \mu \mathrm{s})$ for time-gated imaging of tissues in vivo, completely eliminating shorter-lived $(10<\mathrm{ns})$ emission signals from fluorescent proteins or tissue

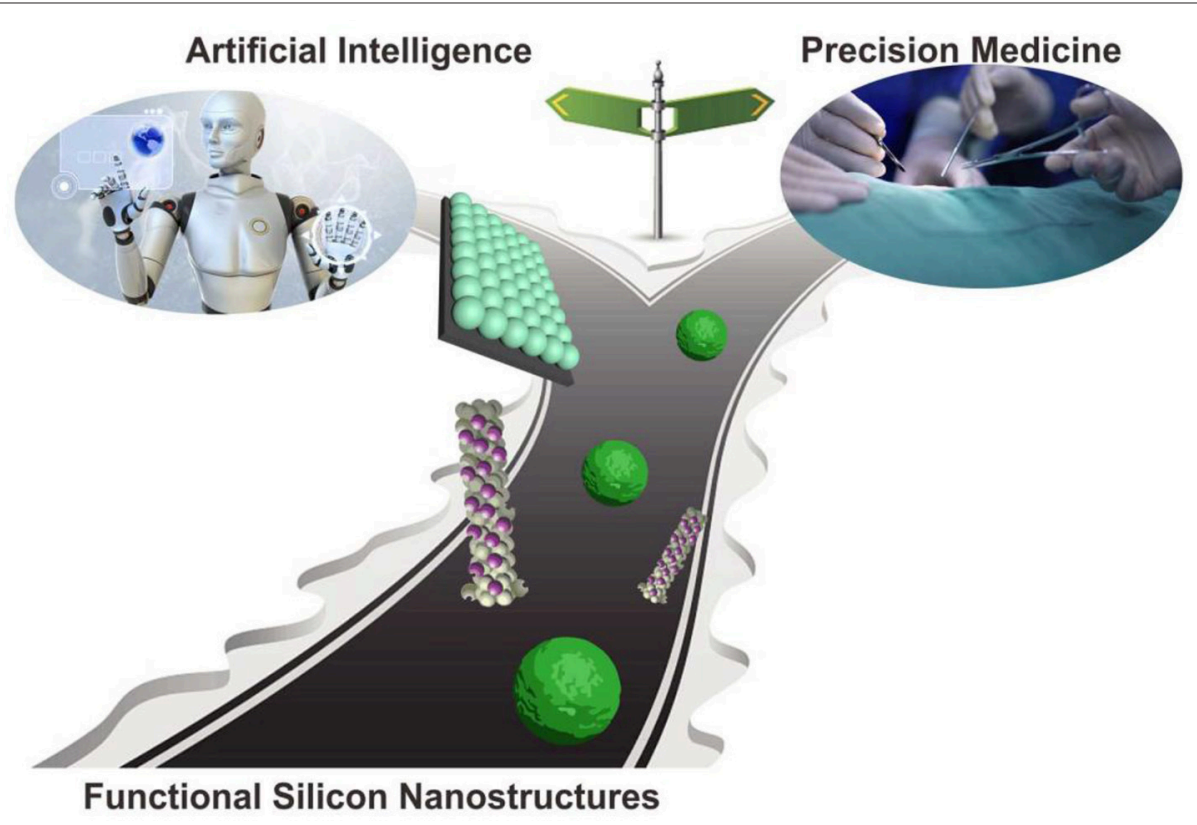

SCHEME 2 | Perspective of silicon nanotechnology in biochemical analysis (Chu et al., 2017, reprinted with permission, Copyright 2017, ACS Publications). 
autofluorescence ( $\mathrm{Gu}$ et al., 2013). In particular, pSiNPsadministrated tumor displayed distinct fluorescence, whereas autofluorescence of normal tissue and short-lived fluorescence of mCherry-expressing tumor were completely removed in the TG image. Later, Tu et al. further demonstrated the fluorescence signals of SiNPs with long photoluminescence lifetimes of ca. $25 \mu$ s could be separated with shorted-lived fluorescein isothiocyanate (FITC) by using TG confocal fluorescence imaging regardless of their overlapped photoluminescence spectra (Tu et al., 2017). Typically, as shown in Figure 2C, co-cultured SKOV3 (human ovarian carcinoma cells) and A431 (human epidermoid carcinoma cells) cancer cells immunostained by functionalized SiNPs and FITC could not be separate from each other in fluorescence imaging and short-lived component, whereas only SiNPs-labeled SKOV3 cells exhibited fluorescence signals in long-lived component. These demonstrations imply that long-lived SiNPs-based TG imaging technique has great potential for high-contrast and high-sensitivity optical imaging, such as precise discernment of tumor margins during surgery without disturbing adjacent normal tissues with background autofluorescence or interfering chromophores with short fluorescence lifetimes.

\section{CONCLUSION AND PERSPECTIVE}

In conclusion, past several years have witnessed considerable progresses in the fabrication of silicon nanomaterials and their applications in biochemical analysis. Several economic and facile synthetic strategies have been developed for the preparation of strong fluorescent SiNPs with controllable colors in facile and large-quantity manners. Meanwhile, effective methods of surface modification have been reported to further improve optical properties and aqueous dispersibility of SiNPs. Besides the zero-dimensional fluorescent SiNPs, onedimensional fluorescent silicon nanostructures (e.g., SiNRs and SiNSs) have been fabricated. Current challenge remains that the exact photoluminescence mechanism of fluorescent silicon nanostructures is controversially to some extent, which requires thorough elucidation in the future.

In terms of sensing applications, benefiting from superior optical properties (i.e., strong and stable fluorescence), SiNPs have been designed as diversified fluorescent sensors, and silicon nanohybrids-based substrates have been employed for the fabrication of high-performance SERS sensors. Such high-quality sensing platform featuring high sensitivity, favorable specificity, and excellent reproducibility, is extremely suitable for the

\section{REFERENCES}

Atkins, T. M., Thibert, A., Larsen, D. S., Dey, S., Browning, N. D., and Kauzlarich, S. M. (2011). Femtosecond ligand/core dynamics of microwaveassisted synthesized silicon quantum dots in aqueous solution. J. Am. Chem. Soc. 133, 20664-20667. doi: 10.1021/ja207344u

Ban, R., Zheng, F. F., and Zhang, J. R. (2015). A highly sensitive fluorescence assay for 2, 4, 6-trinitrotoluene using amine-capped silicon quantum dots as a probe. Anal. Methods 7, 1732-1737. doi: 10.1039/C4AY0 2729A determination and analysis of chemical reagents and biological species in reliable and sensitive manners. Notwithstanding, it is worth pointing out that current sensing applications are mostly limited in the lab research, extensive effort is therefore required to improve the consolidated feasibility of the silicon-based sensors for measurement and analysis of practical samples. On the other hand, silicon nanomaterialbased SERS database featuring superior SERS enhancement and better reproducibility can be collected and served as input data for SERS spectra-based artificial intelligence sensing application, potentially assisting artificial intelligence (AI) to make decisions in sensitive and reliable manners. For bioimaging fields, by the virtue of the superior optical properties (i.e., robust fluorescence coupled with ultrahigh photostability), SiNPs have been employed as novel promising fluorescent nanoprobes, which enable monitoring dynamic biological procedures in long-term and real-time manners. Despite of these exciting research advances on the exploitation of SiNPs for biological imaging analysis, deep investigations are still necessary to explore the potential feasibility for clinic cancer treatment (e.g., intraoperative imaging and surgical excision of sentinel lymph nodes).

We believe that accompanied by deepening understanding of the above-mentioned challenges, the silicon-based bioimaging and nanosensors would raise new perspectives for various biochemical analysis studies (Scheme 2), and show great potentiality for extensive practical applications in biochemical analytical and sensors fields (e.g., artificial intelligence and precision medicine).

\section{AUTHOR CONTRIBUTIONS}

$\mathrm{XJ}, \mathrm{HW}, \mathrm{BS}$, and $\mathrm{BC}$ reviewed literatures and wrote the manuscript text. $\mathrm{YH}$ reviewed the article.

\section{ACKNOWLEDGMENTS}

We appreciate financial support from the National Basic Research Program of China (973 Program, 2013CB934400), the National Natural Science Foundation of China (61361160412, 31400860, 21575096, and 21605109), The Natural Science Foundation of Jiangsu Province of China (BK20170061), and a project funded by the Priority Academic Program Development of Jiangsu Higher Education Institutions (PAPD), 111 Project as well as Collaborative Innovation Center of Suzhou Nano Science and Technology (NANO-CIC).

Cao, Z., Peng, F., Hu, Z., Chu, B., Zhong, Y., Su, Y., et al. (2017). In vitro cellular behaviors and toxicity assays of small-sized fluorescent silicon nanoparticles. Nanoscale 9, 7602-7611. doi: 10.1039/C7NR0 0530J

Chen, N., Ding, P., Shi, Y., Jin, T., Su, Y., Wang, H., et al. (2017). Portable and reliable surface-enhanced Raman scattering silicon chip for signal-on detection of trace trinitrotoluene explosive in real systems. Anal. Chem. 89, 5072-5078. doi: 10.1021/acs.analchem.7b00521

Chiappini, C., De Rosa, E., Martinez, J. O., Liu, X., Steele, J., Stevens, M. M., et al. (2015). Biodegradable silicon nanoneedles delivering nucleic acids 
intracellularly induce localized in vivo neovascularization. Nat. Mater. 14, 532-539. doi: 10.1038/nmat4249

Chu, B. B., Song, B., Ji, X. Y., Su, Y. Y., Wang, H. Y., and He, Y. (2017). Fluorescent silicon nanorods-based ratiometric sensors for long-term and real-time measurements of intracellular $\mathrm{pH}$ in live cells. Anal. Chem. 88, 12152-12159. doi: 10.1021/acs.analchem.7b02791

Chu, B., Wang, H., Song, B., Peng, F., Su, Y. Y., and He, Y. (2016). Fluorescent and photostable silicon nanoparticles sensors for real-time and long-term intracellular pH measurement in live cells. Anal. Chem. 88, 9235-9242. doi: 10.1021/acs.analchem.6b02488

Dasog, M., Kehrle, J., Rieger, B., and Veinot, J. G. (2016). Silicon nanocrystals and silicon-polymer hybrids: synthesis, surface engineering, and applications. Angew. Chem. Int. Ed. 55, 2322-2339. doi: 10.1002/anie.201506065

Erogbogbo, F., Yong, K. T., Roy, I., Hu, R., Law, W. C., Zhao, W., et al. (2011). In vivo targeted cancer imaging, sentinel lymph node mapping and multi-channel imaging with biocompatible silicon nanocrystals. ACS Nano 5, 413-423. doi: 10.1021/nn1018945

Gao, A., Zou, N., Dai, P., Lu, N., Li, T., Wang, Y., et al. (2013). Signal-to-noise ratio enhancement of silicon nanowires biosensor with rolling circle amplification. Nano Lett. 13, 4123-4130. doi: 10.1021/nl401628y

Gonzalez, C. M., Iqbal, M., Dasog, M., Piercey, D. G., Lockwood, R., Klapötke, T. M., et al. (2014). Detection of high-energy compounds using photoluminescent silicon nanocrystal paper based sensors. Nanoscale 6, 2608-2612. doi: 10.1039/C3NR06271F

Gu, L., Hall, D. J., Qin, Z., Anglin, E., Joo, J., Mooney, D. J., et al. (2013). In vivo time-gated fluorescence imaging with biodegradable luminescent porous silicon nanoparticles. Nat. Commun. 4:2326. doi: 10.1038/ncomms3326

Holzinger, M., Le Goff, A., and Cosnier, S. (2014). Nanomaterials for biosensing applications. Front. Chem. 2:63. doi: 10.3389/fchem.2014.00063

Ji, X., Peng, F., Zhong, Y., Su, Y., Jiang, X., Song, C., et al. (2015). Highly fluorescent, photostable, and ultrasmall silicon drug nanocarriers for long-term tumor cell tracking and in vivo cancer therapy. Adv. Mater. 27, 1029-1034. doi: 10.1002/adma.201403848

Jiang, X., Jiang, Z., Xu, T., Su, S., Zhong, Y., Peng, F., et al. (2013). Surfaceenhanced Raman scattering-based sensing in vitro: facile and label-free detection of apoptotic cells at the single-cell level. Anal. Chem. 85, 2809-2816. doi: 10.1021/ac303337b

Joo, J., Liu, X., Kotamraju, V. R., Ruoslahti, E., Nam, Y., and Sailor, M. J. (2015). Gated luminescence imaging of silicon nanoparticles. ACS Nano 9, 6233-6241. doi: 10.1021/acsnano.5b01594

Jose, A. R., Sivasankaran, U., Menon, S., and Kumar, K. G. (2016). A silicon nanoparticle based turn off fluorescent sensor for sudan I. Anal. Methods 8, 5701-5706. doi: 10.1039/C6AY01125J

Jung, J. H., Cheon, D. S., Liu, F., Lee, K. B., and Seo, T. S. (2010). A graphene oxide based immune-biosensor for pathogen detection. Angew. Chem. Int. Ed. 49, 5708-5711. doi: 10.1002/anie.201001428

Kim, D., Kang, J., Wang, T., Ryu, H. G., Zuidema, J. M., Joo, J., et al. (2017). Two-photon in vivo imaging with porous silicon nanoparticles. Adv. Mater. 29:1703309. doi: 10.1002/adma.201703309

Knopfmacher, O., Hammock, M. L., Appleton, A. L., Schwartz, G., Mei, J., and Lei, T., et al. (2014). Highly stable organic polymer field-effect transistor sensor for selective detection in the marine environment. Nat. Commun. 5:2954. doi: 10.1038/ncomms3954

Krivitsky, V., Zverzhinetsky, M., and Patolsky, F. (2016). Antigen-dissociation from antibody-modified nanotransistor sensor arrays as a direct biomarker detection method in unprocessed biosample. Nano Lett. 16, 6272-6281. doi: 10.1021/acs.nanolett.6b02584

Lai, C. H., Hütter, J., Hsu, C. W., Tanaka, H., Varela-Aramburu, S., Cola, L., et al. (2016). Analysis of carbohydrate-carbohydrate interactions using sugarfunctionalized silicon nanoparticles for cell imaging. Nano Lett. 16, 807-811. doi: 10.1021/acs.nanolett.5b04984

Liu, X. K., Zhang, Y. H., Yu, T., Qiao, X. S., Gresback, R., Pi, X. D., et al. (2016). Optimum quantum yield of the light emission from 2 to $10 \mathrm{~nm}$ hydrosilylated silicon quantum dots. Part. Part. Syst. Charact. 33, 44-52. doi: 10.1002/ppsc.201500148

Lu, N., Gao, A., Dai, P., Song, S., Fan, C., Wang, Y., et al. (2014). CMOScompatible silicon nanowire field-effect transistors for ultrasensitive and labelfree microRNAs sensing. Small 10, 2022-2028. doi: 10.1002/smll.201302990
Lu, X., Hessel, C., Yu, Y., Bogart, T., and Korgel, B. (2013). Colloidal luminescent silicon nanorods. Nano Lett. 13, 3101-3105. doi: 10.1021/nl401802h

Nishimura, H., Ritchie, K., Kasai, R. S., Goto, M., Morone, N., Sugimura, H., et al. (2013). Biocompatible fluorescent silicon nanocrystals for singlemolecule tracking and fluorescence imaging. J. Cell Biol. 202, 967-983. doi: $10.1083 /$ jcb.201301053

Pang, J. Y., Su, Y. Y., Zhong, Y. L., Peng, F., Song, B., and He, Y. (2016). Fluorescent silicon nanoparticle-based gene carriers featuring strong photostability and feeble cytotoxicity. Nano Res. 9, 3027-3037. doi: 10.1007/s12274-0161185-8

Park, J. H., Gu, L., von Maltzahn, G., Ruoslahti, E., Bhatia, S. N., and Sailor, M. J. (2009). Biodegradable luminescent porous silicon nanoparticles for in vivo applications. Nat. Mater. 8, 331-336. doi: 10.1038/nmat2398

Park, N. M., Choi, C. J., Seong, T. Y., and Park, S. J. (2001). Quantum confinement in amorphous silicon quantum dots embedded in silicon nitride. Phys. Rev. Lett. 86, 1355-1357. doi: 10.1103/PhysRevLett.86.1355

Peng, F., Su, Y., Zhong, Y., Fan, C. H., Lee, S. T., and He, Y. (2014). Silicon nanomaterials platform for bioimaging, biosensing, and cancer therapy. Acc. Chem. Res. 47, 612-623. doi: 10.1021/ar400221g

Phillips, E., Penate-Medina, O., Zanzonico, P. B., Carvajal, R. D., Mohan, P., Ye, Y., et al. (2014). Clinical translation of an ultrasmall inorganic optical-PET imaging nanoparticle probe. Sci. Trans. Med. 6, 260 ral49. doi: 10.1126/scitranslmed.3009524

Qing, Q., Jiang, Z., Xu, L., Gao, R., Mai, L., and Lieber, C. M. (2014). Free-standing kinked nanowire transistor probes for targeted intracellular recording in three dimensions. Nat. Nanotechnol. 9, 142-147. doi: 10.1038/nnano.2013.273

Shabaev, A., Hellberg, C. S., and Efros, A. L. (2013). Efficiency of multiexciton generation in colloidal nanostructures. Acc. Chem. Res. 46, 1242-1251. doi: $10.1021 /$ ar300283j

Shehada, N., Brönstrup, G., Funka, K., Christiansen, S., Leja, M., and Haick, H. (2014). Ultrasensitive silicon nanowire for real-world gas sensing: noninvasive diagnosis of cancer from breath volatolome. Nano Lett. 15, 1288-1295. doi: $10.1021 / \mathrm{nl} 504482 \mathrm{t}$

Shi, H., Chen, N., Su, Y., Wang, H., and He, Y. (2017). Reusable siliconbased surface-enhanced Raman scattering ratiometric aptasensor with high sensitivity, specificity and reproducibility. Anal. Chem. 89, 10279-10285. doi: 10.1021/acs.analchem.7b01881

Shi, Y., Wang, H., Jiang, X., Sun, B., Song, B., Su, Y., et al. (2016). Ultrasensitive, specific, recyclable, and reproducible detection of lead ions in real systems through a polyadenine-assisted, surface-enhanced, Raman scattering silicon chip. Anal. Chem. 88, 3723-3729. doi: 10.1021/acs.analchem.5b04551

Shiohara, A., Hanada, S., Prabakar, S., Fujioka, K., Lim, T. H., Yamamoto, K., et al. (2010). Chemical reactions on surface molecules attached to silicon quantum dots. J. Am. Chem. Soc. 132, 248-253. doi: 10.1021/ja906501v

Shiohara, A., Prabakar, S., Faramus, A., Hsu, C. Y., Lai, P. S., Northcotea, P. T., et al. (2011). Sized controlled synthesis, purification, and cell studies with silicon quantum dots. Nanoscale 3, 3364-3370. doi: 10.1039/c1nr10458f

Song, B., Wang, H., Zhong, Y., Chu, B., Su, Y., and He, Y. (2018). Fluorescent and magnetic anti-counterfeiting realized by biocompatible multifunctional silicon nanoshuttle-based security ink. Nanoscale 10, 1617-1621. doi: 10.1039/C7NR06337G

Song, B., Zhong, Y., Wang, H., Su, Y., and He, Y. (2017). One-dimensional silicon nanoshuttles simultaneously featuring fluorescent and magnetic properties. Chem. Commun. 53, 6957-6960. doi: 10.1039/C7CC02964K

Song, B., Zhong, Y., Wu, S., Chu, B., Su, Y., and He, Y. (2016). One-dimensional fluorescent silicon nanorods featuring ultrahigh photostability, favorable biocompatibility, and excitation wavelength-dependent emission spectra. J. Am. Chem. Soc. 138, 4824-4831. doi: 10.1021/jacs.6b00479

Song, C., Zhong, Y. L., Jiang, X., Peng, F., Lu, Y., Ji, X., et al. (2015). Peptide-conjugated fluorescent silicon nanoparticles enabling simultaneous tracking and specific destruction of cancer cells. Anal. Chem. 87, 6718-6723. doi: 10.1021/acs.analchem.5b00853

Sun, B., Jiang, X., Wang, H., Song, B., Zhu, Y., Wang, H., et al. (2015). A surfaceenhancement Raman scattering sensing strategy for discriminating trace mercuric ion (II) from real water samples in sensitive, specific, recyclable and reproducible manners. Anal. Chem. 87, 1250-1256. doi: 10.1021/ac503939d

Tilmaciu, C., and Morris, M. C. (2015). Carbon nanotube biosensors. Front. Chem. 3:59. doi: $10.3389 /$ fchem. 2015.00059 
Tu, C. C., Awasthi, K., Chen, K., Lin, C., Hamada, M., Ohta, N., et al. (2017). Time-gated imaging on live cancer cells using silicon quantum dot nanoparticles with long-lived fluorescence. ACS Photonics 4, 1306-1315. doi: 10.1021/acsphotonics.7b00188

Wang, H., Jiang, X., and He, Y. (2016). Highly sensitive and reproducible siliconbased surface-enhanced Raman scattering sensors for real applications. Analyst 141, 5010-5019. doi: 10.1039/C6AN01251E

Wang, H., Zhou, Y., Jiang, X., Sun, B., Zhu, Y., Wang, H., et al. (2015). Simultaneous capture, detection, and inactivation of bacteria as enabled by a surface-enhanced Raman scattering multifunctional chip. Angew. Chem. Int. Ed. 54, 5132-5136. doi: 10.1002/anie.2014 12294

Wang, S. Y., Jiang, X. X., Xu, T. T., Wei, X. P., Lee, S. T., and He, Y. (2014). Reactive ion etching-assisted surface-enhanced Raman scattering measurements on the single nanoparticle level. Appl. Phy. Lett. 104, 243104. doi: 10.1063/1.48 84060

Wang, Y., Wang, T., Da, P., Xu, M., Wu, H., and Zheng, G. (2013). Silicon nanowires for biosensing, energy storage, and conversion. Adv. Mater. 25, 5177-5195. doi: 10.1002/adma.201301943

Wilson, W. L., Szajowski, P. F., and Brus, L. (1993). Quantum confinement in size-selected, surface oxidized silicon nanocrystals. Science 262, 1242-1244. doi: $10.1126 /$ science. 262.5137 .1242

Wipf, M., Stoop, R. L., Tarasov, A., Bedner, K., Fu, W., Wright, I. A., et al. (2013). Selective sodium sensing with gold-coated silicon nanowire field-effect transistors in a differential setup. ACS Nano 7, 5978-5983. doi: $10.1021 / \mathrm{nn} 401678 \mathrm{u}$

Wu, K., Du, Y., Tang, H., Chen, Z., and Lian, T. (2015). Efficient extraction of trapped holes from colloidal CdS nanorods. J. Am. Chem. Soc. 137, 10224-10230. doi: 10.1021/jacs. 5 b0 4564

Wu, S., Zhong, Y., Zhou, Y., Song, B., Chu, B., Ji, X., et al. (2015). Biomimetic preparation and dual-color bioimaging of fluorescent silicon nanoparticles. J. Am. Chem. Soc. 137, 14726-14732. doi: 10.1021/jacs.5b08685

Wu, Y., Zhong, Y., Chu, B., Sun, B., Song, B., Wu, S., et al. (2016). Plant-derived fluorescent silicon nanoparticles featuring excitation wavelength-dependent fluorescence spectra for anti-counterfeiting application. Chem. Commun. 52, 7047-7050. doi: 10.1039/C6CC02872A

Xie, C., Liu, J., Fu, T. M., Dai, X., Zhou, W., and Lieber, C. M. (2015). Threedimensional macroporous nanoelectronic networks as minimally invasive brain probes. Nat. Mater. 14, 1286-1292. doi: 10.1038/nmat4427
Yi, Y., Deng, J., Zhang, Y., Li, H., and Yao, S. (2013a). Label-free Si quantum dots as photoluminescence probes for glucose detection. Chem. Commun. 49, 612-614. doi: 10.1039/C2CC36282A

Yi, Y., Zhu, G., Liu, C., Huang, Y., Zhang, Y., Li, H., et al. (2013b). A labelfree silicon quantum dots-based photoluminescence sensor for ultrasensitive detection of pesticides. Anal. Chem. 85, 11464-11470. doi: 10.1021/ac403257p

Zheng, J. Y., Yan, Y., Wang, X., Zhao, Y., Huang, J., and Yao, J. (2012). Wireon-wire growth of fluorescent organic heterojunctions. J. Am. Chem. Soc. 134, 2880-2883. doi: 10.1021/ja209815f

Zhong, Y., Peng, F., Bao, F., Wang, S., Ji, X., Yang, L., et al. (2013). Largescale aqueous synthesis of fluorescent and biocompatible silicon nanoparticles and their use as highly photostable biological probes. J. Am. Chem. Soc. 135, 8350-8356. doi: 10.1021/ja4026227

Zhong, Y., Sun, X., Wang, S., Peng, F., Bao, F., Su, Y., et al. (2015). Facile, large-quantity synthesis of stable, tunable-color silicon nanoparticles and their application for long-term cellular imaging. ACS Nano 9, 5958-5967. doi: 10.1021/acsnano.5b00683

Zhou, J., Yang, Y., and Zhang, C. Y. (2015). Toward biocompatible semiconductor quantum dots: from biosynthesis and bioconjugation to biomedical application. Chem. Rev. 115, 11669-11717. doi: 10.1021/acs.chemrev.5b00049

Zhou, Y. F., Zhang, Y., Zhong, Y. L., Fu, R., Wu, S. C., Wang, Q., et al. (2017) The in vivo targeted molecular imaging of fluorescent silicon nanoparticles in Caenorhabditis elegans. Nano Res. doi: 10.1007/s12274-017-1677-1. [Epub ahead of print].

Zhu, Y., Jiang, X., Wang, H., Wang, H., Su, Y., and He, Y. (2015). A poly Adeninemediated assembly strategy for designing surface-enhanced resonance Raman scattering substrates in controllable manners. Anal. Chem. 87, 6631-6638. doi: 10.1021/acs.analchem.5b00676

Conflict of Interest Statement: The authors declare that the research was conducted in the absence of any commercial or financial relationships that could be construed as a potential conflict of interest.

Copyright (c) $2018 \mathrm{Ji}$, Wang, Song, Chu and He. This is an open-access article distributed under the terms of the Creative Commons Attribution License (CC $B Y)$. The use, distribution or reproduction in other forums is permitted, provided the original author(s) and the copyright owner are credited and that the original publication in this journal is cited, in accordance with accepted academic practice. No use, distribution or reproduction is permitted which does not comply with these terms. 